\title{
Improving Efficiency in Separating Blood Vessels from Retinal Images with Deep Learning Techniques
}

\author{
Gotlur Karuna, Kantedi Prashanth, G.Kalpana
}

\begin{abstract}
Retinal vessels ID means to isolate the distinctive retinal configuration issues, either wide or restricted from fundus picture foundation, for example, optic circle, macula, and unusual sores. Retinal vessels recognizable proof investigations are drawing in increasingly more consideration today because of pivotal data contained in structure which is helpful for the identification and analysis of an assortment of retinal pathologies included yet not restricted to: Diabetic Retinopathy (DR), glaucoma, hypertension, and Age-related Macular Degeneration (AMD). With the advancement of right around two decades, the inventive methodologies applying PC supported systems for portioning retinal vessels winding up increasingly significant and coming nearer. Various kinds of retinal vessels segmentation strategies discussed by using Deep Learning methods. At that point, the pre-processing activities and the best in class strategies for retinal vessels distinguishing proof are presented.
\end{abstract}

Index Terms: classification, deep learning, feature learning, retina, vessel segmentation.

\section{INTRODUCTION}

Retinal structure embroils imperative data helps to identifying and diagnoses an assortment of retinal pathology that are related to the irregular varieties in retinal vascular structure. In this way, vital demonstrative esteem a rule, (vessels structure-like) segmentation involve an astounding spot in medicinal picture separation field [1, 2, 3, 4] retinal vessels. Segmented parts has a place with this classification where an expansive assortment of calculations and techniques have been produced and executed for programmed ID [5].

This paper work mainly aimed to present a detailed survey that covers and sorts early and ongoing writing strategies, systems, with the significant spotlight on the location and different parts of retinal structures existent in two dimensional retinal fundus pictures. Furthermore, our audit covers the hypothetical premise behind every classification just as the related focal points and constraints.

Revised Version Manuscript Received on 16 September, 2019

Gotlur Karuna, Computer Science and Engineering, GRIET, Hyderabad, India.

Kandeti Prashanth, Computer Science and Engineering, GRIET, Hyderabad, India. India.
The dimensions of deep Neural Networks (NN) originate from a few advancements. Exchange elements of units in customary NNs are regularly pressing capacities, with subsidiaries near zero all over the place. This prompts lessening slope in preparing the back proliferated mistakes rapidly decline with each system layer, rendering preparing insufficient or horrendously moderate. Interestingly, the move capacities utilized in deep CNNs [8], most remarkably the amending direct units [5], don't evaporate in boundaries thus permit viable preparing of networks with many layers [4]. Also, Deep Learning carried with it new strategies for boosting system heartiness like dropout [6], where arbitrarily chosen units are briefly handicapped. This powers a system to shape weight setups that give right yields irrespective of some picture highlights are missing thus enhances speculation.

\section{RELATED WORK}

\section{A. Retinal Image Processing}

Retina vessel distinguishing proof and extraction faces numerous difficulties that are demonstrated as specified in [7]. Right off the bat, the retinal vessels scope of shading power run from short of what one pixel up to in excess of five pixels in the retinal picture [8], as appeared in cry, which requires an ID strategy with high adaptability as shown in figure1.

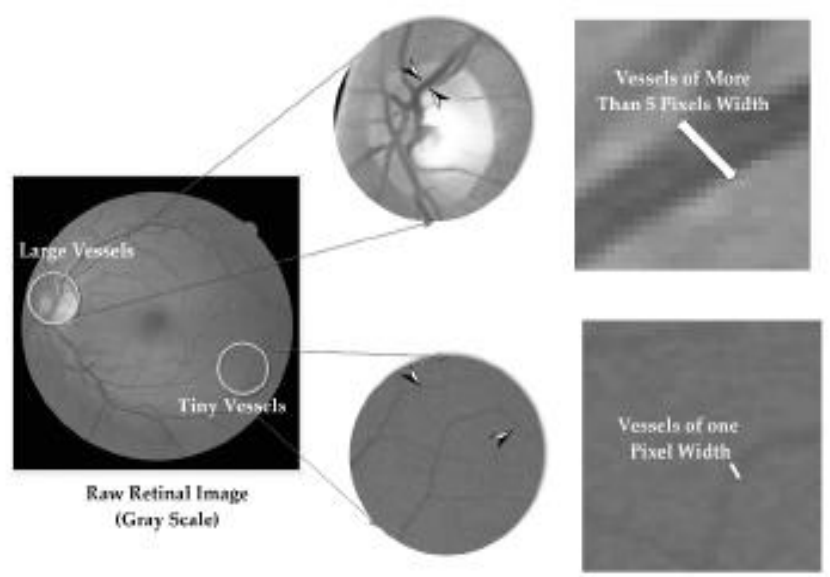

Fig 1: Pixel width difference of retinal vessels.

Published By Blue Eyes Intelligence Engineering 
To expound this test, a scrap of MATLAB® code has been produced for purpose of dark dimensions substitution in retinal picture; the diverse dim dimensions of a crude retinal picture have been supplanted by shading ones. It tends to be noticed that numerous retinal vessels, either massive or modest ones, take a related foundation shading powers. This uncovers the wide scope of hues that might be distinguishing proof procedure more entangled instead of that found in other recognizable proof issues [9].

\section{B. Retinal Vessels Segmentation Techniques}

For the most part, with respect to segmentation issue, there exist the same number of methods and computations as there are explicit cases and circumstances [8]. Among them, segmentation instruments utilized for therapeutic purposes when all is said in done and for retinal separation specifically [11]. These techniques share basic stages: pre-handling, preparing and post-preparing. In this survey, we have ordered the secured papers dependent on the calculation [11] or procedure utilized in the preparing, yielding six noteworthy classifications: 1 . bit based systems; 3 . Scientific morphology-based 4. Multi-scale 7. Machine learning.

Affectability and particularity speak to the most regularly used measurements in therapeutic research; the higher the explicitness and affectability esteems, the better finding [8, 9]. The affectability mirrors the ability of the computation to recognize the vessels pixels, though the particularity decides investigate; the higher the explicitness and affectability esteems, the better analysis. The affectability mirrors the capacity of calculation to differentiate vessels' pixels, though particularity determines the capacity of calculation to recognize non-vessel pixels. Affectability and Specificity speak to highlights of the calculation and are related with the accurate metric in numerous therapeutic picture preparing fields, including retinal vessel segmentation.

\section{Kernel-Based Techniques}

The fundamental thought of piece based systems (additionally called coordinated sifting based) procedures is to contrast the pixels' force varieties along and the cross-segment profile of retinal vessel with a prefigure layout fills in as a bit [9]. In this way, most run of the mill coordinated channel based systems discriminate the retinal vessels to applying a coordinated channel piece on the first dark retinal picture pursued by a thresholding step.

Retinal vessel profiling is a type of grouping [10]. On account of vessels identification and utilized to make guide for procedure discovery, makes ready for vessels extraction of area developing or sifting based methodologies. As a rule, retinal vascular coordinated parts can fall of two noteworthy classifications: Gaussian molded or non-Gaussian formed.

D. Vessel Tracking/Tracing Techniques
In following strategies is not for seed focuses (beginning stages of following procedure) to be located at the focal point of retinal vessels, Chutatape et al., have separated seed
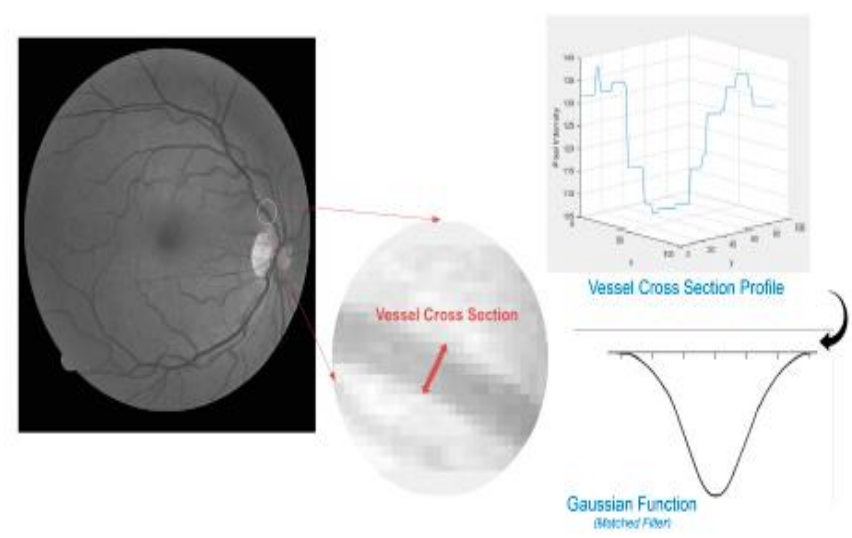

Fig 2: Cross-section intensity profile

focuses from the circuit of the optic circle, at that point the focuses of vessels were followed utilizing an all-inclusive Kalman channel [7]. A semi-oval was characterized around the optic circle as a hunting locale down beginning stages of vascular structure, here focuses were chosen on the semi-oval, vessel following occurred dependent on Bayesian hypothesis.

The following execution was tried by means of DRIVE dataset.

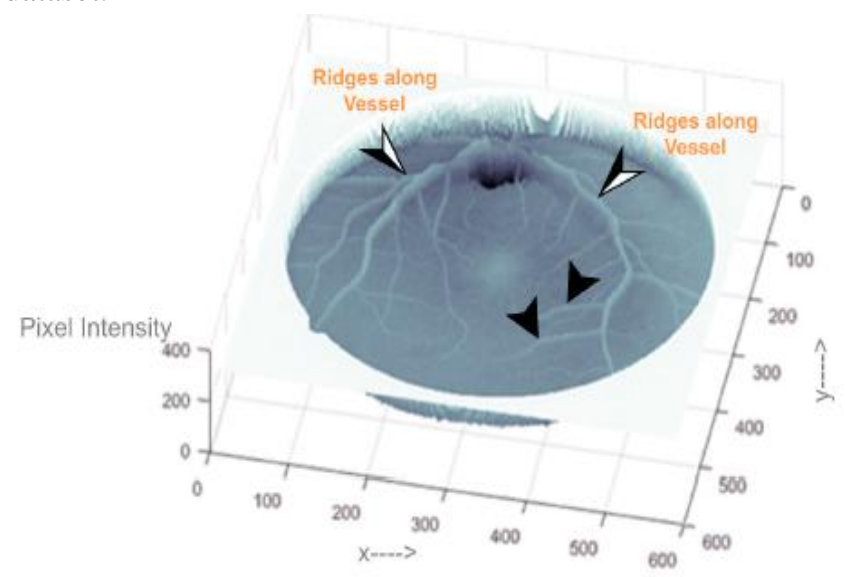

Fig 3: Representation of ridges along retinal tree.

Making utilization of scientific diagram hypothesis, De et al. [8] planned a novel strategy to extricate the retinal structure. Their strategy was assembled dependent on associating the following issue and the digraph grid woods hypothesis in arithmetical diagram hypothesis with an essential objective to address the vessel traverse issue.

Here system is prepared by using two primary stages. The initial step is utilized to develop the digraph portrayal, which empowers following undertaking to give a role as digraph-based name spread utilizing Matrix-woodland hypothesis. 


\section{E. Machine-Learning Techniques}

Machine learning tends to the issues where, for each info $\mathrm{x}$, there is a comparing watched yield $y$, while, on account of the last two classifications, this correspondence can't be found because of the absence of information. Unsupervised learning investigates fascinating illustrations with regards to the information without a requirement for express supervision [8,11]. Fortification learning expects that the elements of the framework under thought pursue a specific class of model.

A tale conspire dependent on blend of hereditary calculation and fluffy c-implies clustering algorithm. In pre-handling, the crude retinal picture was extricated and improved by histogram evening out. The highlights, containing information got in the principal organize, were bunched by means of fluffy c-implies calculation related to hereditary calculation, where, right off the bat, the hereditary calculation is utilized to acquire the rough arrangement of the worldwide ideal arrangement; and, furthermore, the surmised arrangement was utilized as starting estimation of the fluffy c-implies calculation.

\section{RESULTS}

The present work depicted the comparative results of various retinal segmentation techniques such as kernel based, vessel tracking, multi-scale, model-based, image based and deep learning techniques as shown in below Table1.

Table 1: different kinds of retinal segmentation techniques

\begin{tabular}{|c|c|c|c|c|}
\hline S.No & Segmentation technique & Type & $\begin{array}{l}\text { Performance } \\
\text { measure }\end{array}$ & $\begin{array}{l}\text { Validation } \\
\text { dataset }\end{array}$ \\
\hline 1. & $\begin{array}{l}\text { Two-dimensional Gaussian matched } \\
\text { filter entropy }\end{array}$ & Kernel based & - & - \\
\hline 2. & $\begin{array}{l}\text { Gaussian matched filter with adaptive } \\
\text { thresholding }\end{array}$ & Kernel based & - & STARE \\
\hline 3. & $\begin{array}{l}\text { Improved t-dimensional Gaussian } \\
\text { matched }\end{array}$ & Kernel based & $\begin{array}{l}\text { Accuracy, specificity, } \\
\text { sensitivity }\end{array}$ & DRIVE, STARE \\
\hline 4. & Vessel tracking by Kalman filter & Vessel tracking & - & - \\
\hline 5. & $\begin{array}{l}\text { Vessel tracking using mathematical } \\
\text { graph theory. }\end{array}$ & Vessel tracking & False Positive Rate & DRIVE, STARE \\
\hline 6. & Gaussian pyramid multi-scaling. & Multi-scaling & $\begin{array}{l}\text { Accuracy, specificity, } \\
\text { sensitivity }\end{array}$ & DRIVE, STARE \\
\hline 7. & Morphological Angular scale space & Morphologicalbased & Mean Square Error & Drive \\
\hline 8. & Back propagation ANN & Machine Learning & sensitivity & - \\
\hline 9. & Deep Convolutional ANNs. & Deep Learning & Recall, precision & DRIVE, STARE \\
\hline 10. & Deep Convolutional ANNs. & Deep Learning & accuracy & DRIVE, STARE \\
\hline
\end{tabular}

Most widely used data sets for retinal vessels segmentation techniques are Digital Retinal Image for Vessel Extraction (DRIVE), Structuring Analysis of the Retina (STARE). The metrics used for computation are precision, accuracy, specificity, false positive rate and sensitivity and calculated by given below formulas.

$$
\text { Precision }=T P /(T P+F P)
$$

$$
\text { Accuracy }=(T P+T N) /(T P+F N+F P+T N)
$$

\author{
Specificity $=T N /(T N+F P)$ \\ Recall or sensitivity $=T P /(T P+F N)$
}

\title{
IV. CONCLUSION
}

The current retinal vein segmentation philosophies are ordered, and depicted in the last area. We talked about different procedures applied in these segmentation techniques for the separation of veins in retinal picture and look at execution consequence of the strategies. These philosophies were assessed utilizing freely accessible datasets. Different retinal vessels segmentation techniques pursue comparable strategies: Preparing step speaks to the core of calculation, where the distinctive procedures sorted in last segment are utilized. At long last, in the post-preparing step, the underlying portioned picture experiences ventures of smoothing and edge protecting and improvement.

\section{REFERENCES}

1. Lesage, D. Angelini, E.D. Bloch, I. and Funka-Lea, G. "A review of three dimensional vessel lumen segmentation techniques - Models, features, extraction schemes". Medical Image Analysis. 2009, 13, 819-845.

2. Kirbas, C.; Quek, F. "A brief review of vessel extraction techniques, algorithms". ACM Computational Survey. 36, 81-121, CSUR 2004,

3. Kirbas, C.; F.K, Quek ; "Vessel extraction techniques and algorithms: A survey" presented in the Third IEEE conference on Bioinformatics and Bioengineering, Bethesda, MD, USA, 12 March 2003; pp. 238-245.

4. Suri, J.S.; Laxminarayan, S., Liu, K.; Reden, L.; “A review on MR vascular image processing approaches" Part II. IEEE Transactions. Information Technology Biomed. 2002, 6, 338-350.

5. Fraz, M.M.; Barman, S.A., Uyyanonvara, B.; Hoppe, A,; Rudnicka, Remagnino, P.; Owen, C.G.; "Blood vessel segmentation methodologies in retinal images: A survey". Pg no: 108, 407-433, Computational Methods Program Biomedical 2012.

6. Srinidhi, C.L.; Aparna, P.; Rajan, J. Recent Advancements in Retinal Vessel Segmentation. J. Med. Syst. 2017, 41, 70.

7. Dash, J.; Bhoi, N."A detailed survey on Blood Vessel Detection approaches in Retinal Images. In Proceedings of the 2015 International Conference on Computational Intelligence and Networks, Bhubaneshwar, India, 12-13 January 2015; pp. 166-171.

8. Kolb, H. Simple Anatomy of the Retina, 2012. Available online: http://webvision.med.utah.edu/book/parti-foundations/simple-anato my-of-the-retina/ (accessed on 22 January 2018).

9. Solouma, N.; Youssef, A.-B.M.; Badr, Y.; Kadah, Y.M. "Real-time retinal tracking for laser treatment planning". Image Processing; SPIE_-The International Society of Optics and Photonics: Bellingham, WA, USA; pp. 1311-1321, Medical Imaging 2001.

10. Gu, L.; Cheng, L. Learning to boost filamentary structure segmentation. In Proceedings of the IEEE International Conference on Computer Vision, Santiago, Chile, 7-13 December 2015; pp. 639-647.

11. Ophthalmic Photographers' Society. Available online: www.opsweb.org (accessed on 22 January 2018). 


\section{AUTHORS PROFILE}

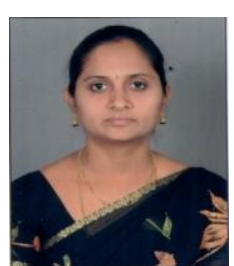

Dr.Gotlur Karuna, Professor in the Department of Computer Science and Engineering at Gokaraju Rangaraju Institute of Engineering and Technology, Hyderabad, Telangana, India. She has completed her Ph.D. in CSE from Jawaharlal Nehru Technological University, Hyderabad, Telangana, India. She has thirteen years of experience in teaching for both undergraduate and post graduate students. She has a life membership of CSI and ISTE. She has published 32 papers in various National and International journals and conferences. Her research areas are Image Processing, Computer Networks, Cryptography and Network Security, Big Data Analytics, Machine Learning.

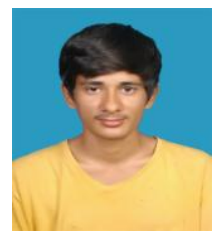

Mr. Kandeti Prashanth is pursuing his post-graduation in the department of computer science and engineering at Gokaraju Rangaraju Institute of Engineering and Technology (GRIET), Hyderabad, Telangana, India.

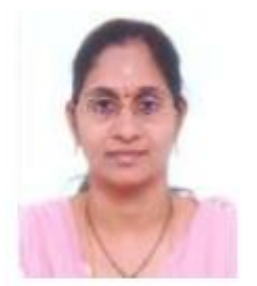

G.Kalpana is an Assistant Professor in the Department of C.S.E, Vidya Jyothi Institute Of Technology, Hyderabad, A.P., India. She has completed her M.Tech. in CSE branch from HITAM, Affiliated Engineering College, Jawaharlal Nehru Technological University, Hyderabad, India in 2010. She did her M.C.A from Annamalai University, Tamil Nadu, India in 2007. She has 13 years experience of teaching for undergraduate students and for post graduate students. She has published/presented 10 papers in National/international journals and Conferences. Her research areas are Image Processing, Data mining and Analytics. 